\title{
Initial consecutive experience of completely portal robotic pulmonary resection with 4 arms
}

\author{
Robert J. Cerfolio, MD, FACS, FCCP, Ayesha S. Bryant, MD, MSPH, Loki Skylizard, MD, and \\ Douglas James Minnich, MD, FACS
}

\begin{abstract}
Background: Many general thoracic surgeons are learning robotic pulmonary resection.
Methods: We retrospectively compared results of completely portal robot lobectomy with 4 arms (CPRL-4) against propensity-matched controls and results after technical changes to CPRL-4.
\end{abstract}

\begin{abstract}
Results: In 14 months, 168 patients underwent robotic pulmonary resection: 7 had metastatic pleural disease, 13 had conversion to open procedures, and 148 had completion robotically (106 lobectomies, 26 wedge resections, 16 segmentectomies). All patients underwent R0 resection and removal of all visible lymph nodes (median of 5 N2, 3 N1 nodal stations, 17 lymph nodes). The 106 patients who underwent CPRL-4 were compared with 318 propensity-matched patients who underwent lobectomy by rib- and nerve-sparing thoracotomy. The robotic group had reduced morbidity $(27 \%$ vs $38 \% ; P=.05)$, lower mortality $(0 \%$ vs $3.1 \% ; P=.11)$, improved mental quality of life (53 vs $40 ; P<.001$ ), and shorter hospital stay ( 2.0 vs 4.0 days; $P=.02$ ). Results of CPRL-4 after technical modifications led to reductions in median operative time ( 3.7 vs 1.9 hours; $P<.001)$ and conversion $(12 / 62$ vs $1 / 106 ; P<.001)$. Technical improvements were addition of fourth robotic arm for retraction, vessel loop to guide the stapler, tumor removal above the diaphragm, and carbon dioxide insufflation.
\end{abstract}

Conclusions: The newly refined CPRL-4 is safe and yields an R0 resection with complete lymph node removal. It has lower morbidity, mortality, shorter hospital stay, and better quality of life than rib- and nerve-sparing thoracotomy. Technical advances are possible to shorten and improve the operation. (J Thorac Cardiovasc Surg 2011;142:740-6)

The increasing use of robotic surgical systems worldwide is undeniable. A recent article in The Wall Street Journal ${ }^{1}$ addressed the problems of learning robotic surgery and also the issue of credentialing for surgeons already in practice. Similarly, an article in The New England Journal of Medicine $^{2}$ in August 2010 estimated the cost of the expanding use of robotic surgery in other specialties. The benefit that any new surgical technique offers should be demonstrated in carefully designed prospective studies; however, the rapid paradigm shift toward minimally invasive surgery, such as video-assisted thoracoscopic surgery (VATS) and

From the Division of Cardiothoracic Surgery, University of Alabama at Birmingham, Birmingham, Ala.

Disclosures: The principle investigator of this study (R.J.C.) has lectured for Intuitive (Sunnyvale, Calif); however, Intuitive had no role in the study design, conduct, data analysis, or drafting of the manuscript or its results. R.J.C. has the following financial relationships: Intuitive, speaker; E Plus Health Care, speaker; Ethicon, speaker and consultant; Neomend, consultant; Millicore, speaker and consultant; Medela, speaker and consultant; Closure/J\&J, consultant; OSI Pharm, speaker; Atrium, consultant and speaker; Oncotech, speaker; Covidien, speaker; and Precision, consultant and speaker. A.S.B. and L.S. have no financial disclosures to report. D.J.M. is a consultant and speaker for SuperDimension.

Received for publication March 21, 2011; revisions received June 1, 2011; accepted for publication July 14, 2011; available ahead of print Aug 16, 2011.

Address for reprints: Robert J. Cerfolio, MD, FACS, FCCP, Professor of Surgery, Chief of Thoracic Surgery, JH Estes Family Endowed Chair for Lung Cancer Research, Division of Cardiothoracic Surgery, University of Alabama at Birmingham, 703 19th St S, ZRB 739, Birmingham, AL 35294 (E-mail: rcerfolio@uab.edu). 0022-5223/\$36.00

Copyright (c) 2011 by The American Association for Thoracic Surgery doi:10.1016/j.jtcvs.2011.07.022 robotic surgery, has occurred without any prospective, randomized trials. The lack of equipoise makes these types of trials unlikely. Robotics is rapidly growing because of the improvements in 3-dimensional visualization, the technical advantages of small-wristed instruments, and the ability to perform an outstanding lymph node dissection. Studies have shown the safety of robotic pulmonary resection, but none have compared outcomes with those of rib-sparing, nerve-sparing thoracotomy. ${ }^{3-6}$ In this study, we report our experience with robotic pulmonary resection with a newly modified technique that features a completely portal (all small incisions) 4-arm robotic operation (CPRL-4) with carbon dioxide insufflation and compare the short-term outcomes with those of patients undergoing resection through a rib- and nerve-sparing thoracotomy. Importantly, this is not a selected series but rather a consecutive series of patients who had clinically apparent resectable non-small cell lung cancer. Therefore patients who underwent VATS lobectomy were not chosen as a comparison group, because it was only offered to selected patients (those with tumors $<4 \mathrm{~cm}$ and without N1 disease).

\section{MATERIALS AND METHODS}

This is a retrospective cohort study of a consecutive series of patients entered into a prospective database during a 14-month period who underwent attempted completely portal robotic pulmonary resection. Patients had apparent resectable lesions that were biopsy proven or were highly suspect for non-small cell lung cancer. All operations were performed by 


\section{Abbreviations and Acronyms \\ CPRL-4 = completely portal robotic lobectomy with 4 robotic arms \\ SF-12 = 12-item Short Form Health Survey \\ VATS $=$ video-assisted thoracoscopic surgery}

a single general thoracic surgeon (R.J.C.) at a single academic center. Almost all patients who in the past would have been offered resection through a thoracotomy (patients who had undergone computed tomographic scanning, integrated positron emission tomographic and computed tomographic scanning, and pulmonary function and cardiac stress testing and who were mediastinal [N2] lymph node negative and had adequate cardiopulmonary reserve, as previously described by us $^{7,8}$ ) were now offered robotic resection. The only patients who were not offered robotic surgery who in the past would have been offered thoracotomy were those who had tumor in the segmental bronchus or more proximal, those who had chest wall involvement that required rib resection, and those who refused a robotic operation. Neither the size of the lesion, its location, the presence of $\mathrm{N} 1$ disease, nor the use of preoperative radiation or chemotherapy contraindicated the offer of robotic pulmonary surgery in this study.

The nerve- and rib-sparing thoracotomy that was used in this series for the matched control patients has been previously described by us elsewhere. ${ }^{9-12}$ It has been corroborated by other centers as less painful than standard thoracotomy. ${ }^{13,14}$ This type of thoracotomy technique was compared with the newly developed completely portal four arm robotic technique (CPRL-4) described in Appendix 1 (Figure 1).

Conversions from a completely portal robotic technique to an open technique were done for several reasons. A time limit of approximately 4 hours (as we learned how to do the operation) was set to prevent patient injury from prolonged anesthesia and to limit both operating room personnel and surgeon frustration. After 4 hours, patients had conversion to open surgery or to VATS if the operation was not near completion. Other indications for opening were bleeding that could not be controlled robotically and the inability to enter the pleural space because of pleural symphysis.

The University of Alabama at Birmingham's institutional review board approved this protocol as well as the prospective database used to collect information for this study. Patient consent was waived for inclusion in this individual study; however, consent was both required and obtained to enter patient data into the prospective database.

\section{Definitions}

Morbidity was defined according to The Society of Thoracic Surgeons database's definitions (version 2.8), with the exception of air leak. Operative mortality was defined as death from any cause within 30 days after surgery or before discharge. The operative time was defined as the time from skin incision until skin closure and thus included all robotic docking and undocking times, along with time spent waiting for frozen-section analysis. A numeric pain score was assessed on a scale ranging from 0 (no pain) to 10 (extreme pain). Quality of life was defined as the subject's functioning and well-being in the physical, psychologic, and social domains in relation to disease and treatment. In this study, the participants' quality of life was measured with the 12-item Short Form Health Survey (SF-12) with supplemental questions about pain control. ${ }^{15}$ The SF-12, a validated shorter alternative to the 36-item survey, consists of a physical component summary and a mental component summary. ${ }^{16-18}$ Subjects completed the SF-12 preoperatively and at both 3 weeks and 4 months postoperatively either at a clinic appointment or by mail. SF-12 scores were computed separately for the physical and mental components of the survey and compared with adjusted values, with 50 representing the average or norm-based

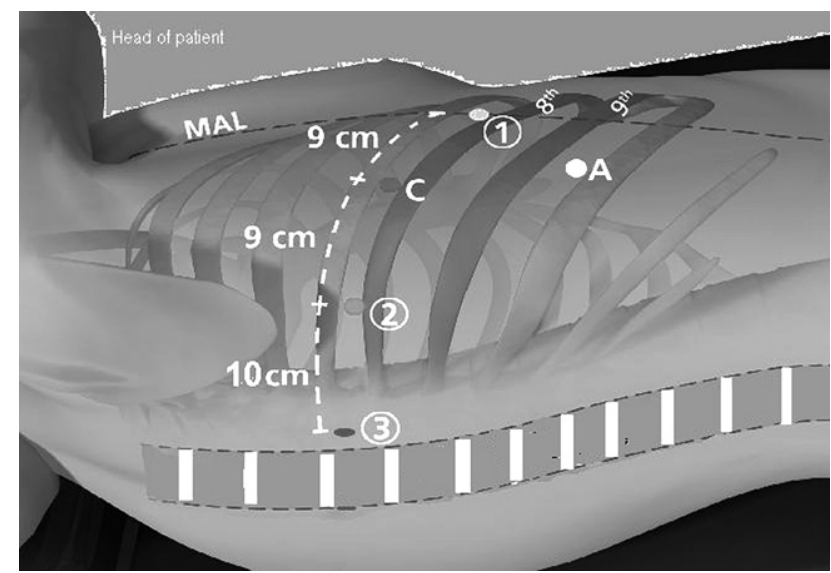

FIGURE 1. The completely portal robotic lobectomy with 4 robotic arms technique developed in this study is shown. It features entering the pleural space with a 5 -mm port anteriorly in the midaxillary line $(M A L)$ over the top of the 7th rib and then using a 5-mm video-assisted thoracoscopic surgery camera to enable the surgeon to make all other incisions on the basis internal anatomy. The circled numbers represent the robotic arms used, $C$ indicates the camera port, and $A$ indicates the 15-mm access port (which can also be placed between the camera and robotic arm 2 if space is not adequate more anteriorly). Note that robotic arm 3 is a $5-\mathrm{mm}$ port, robotic arm 2 is an $8-\mathrm{mm}$ port, the camera can be an 8 - or $12-\mathrm{mm}$ port, depending on the camera used, and robotic arm 1 is a $12-\mathrm{mm}$ port. The area with the dashed lines is the area in which no incisions are made and is the most posterior third of the area between the mid spine and the posterior edge of the scapula.

score. Quality of life information was obtained from patients at both 3 weeks and 4 months after surgery.

\section{Statistical Analysis}

Data were exported from Excel (Microsoft Corp, Redmond, Wash) to SAS v. 9.1 (SAS Institute, Inc, Cary, NC) software. Descriptive statistics were used to estimate the frequencies of the categoric variables and the medians of the continuous variables. Differences between study groups were assessed with the use of 2-sided Fisher's Exact tests and $\chi^{2}$ tests for categoric variables and of independent sample Student $t$ tests for normally distributed continuous variables.

Propensity score analysis was carried out in this study to estimate the probability that a patient might undergo a robotic procedure versus thoracotomy to eliminate the effects of lack of randomization and selection bias. ${ }^{19}$ A logistic regression analysis of several preoperative variables (laterality of tumor, preoperative Eastern Cooperative Oncology Group performance status, sex, age [ \pm 5 years], forced expiratory volume in 1 second $[ \pm 5 \%]$, smoking status, history of neoadjuvant therapy, and size of the tumor resected) was performed to generate a single propensity score for each patient. Patients selected for matching were selected from a database of more than 3000 patients (595 elective lobectomies) operated on between 2006 and 2009 by the same general thoracic surgeon who performed all the robotic operations, CPRL-4 procedures, and thoracotomies in this study (R.J.C.).

\section{RESULTS}

Between February 2010 and April 2011, a total of 168 patients underwent attempted robotic pulmonary resection for clinically staged resectable disease. There were 55 patients 
TABLE 1. Characteristics of the 168 patients in this study and a comparison of the 106 patients who underwent completely portal robotic lobectomy with 4 robotic arms compared with 317 patients (propensity-matched controls) who underwent lobectomy with a rib- and nervesparing thoracotomy

\begin{tabular}{|c|c|c|c|}
\hline & $\begin{array}{l}\text { All patients with attempted } \\
\text { robotic operation }(N=168)\end{array}$ & $\begin{array}{c}\text { Robotic lobectomy }(\mathrm{N}=106, \\
\text { excludes } 13 \text { conversions })\end{array}$ & $\begin{array}{c}\text { Rib-, nerve-sparing } \\
\text { thoracotomy }(\mathrm{N}=\mathbf{3 1 8})\end{array}$ \\
\hline Age* $^{*} \mathrm{y}$, median and range) & $67(21-87)$ & $66(31-85)$ & $66(26-83)$ \\
\hline Male $\operatorname{sex}^{*}$ (no.) & $75(45 \%)$ & $51(48 \%)$ & $150(47 \%)$ \\
\hline \multicolumn{4}{|l|}{ Ethnicity (no.) } \\
\hline White & $146(87 \%)$ & $92(88 \%)$ & $273(86 \%)$ \\
\hline Black & $21(13 \%)$ & $13(12 \%)$ & $44(14 \%)$ \\
\hline Other & $1(<1 \%)$ & $1(1 \%)$ & $1(<1 \%)$ \\
\hline $\begin{array}{l}\text { Current smoking or history* (smoking within } 6 \text { mo } \\
\text { of surgery and }>100 \text { cigarettes in lifetime, no.) }\end{array}$ & $32(19 \%)$ & $18(17 \%)$ & $51(16 \%)$ \\
\hline Forced expiratory volume in $1 \mathrm{~s} *(\%$, median $\pm \mathrm{SD})$ & $83.9 \pm 14.5$ & $84.1 \pm 12.0$ & $85.4 \pm 13$ \\
\hline $\begin{array}{l}\text { Diffusing capacity of lung for carbon } \\
\text { monoxide (adjusted } \%, \text { median } \pm \text { SD) }\end{array}$ & $77.5 \pm 13.5$ & $76.2 \pm 11.4$ & $80.2 \pm 12.8$ \\
\hline $\begin{array}{l}\text { Eastern Cooperative Oncology Group performance } \\
\text { status* (median and range) }\end{array}$ & $1(0-3)$ & $1(0-3)$ & $1(0-3)$ \\
\hline Neoadjuvant therapy* (no.) & $6(4 \%)$ & $5(5 \%)$ & $16(5 \%)$ \\
\hline History of hypertension (no.) & $82(49 \%)$ & $73(69 \%)$ & $191(60 \%)$ \\
\hline History of cardiac disease (no.) & $36(21 \%)$ & $33(32 \%)$ & $89(28 \%)$ \\
\hline History of diabetes mellitus (no.) & $17(10 \%)$ & $16(15 \%)$ & $35(11 \%)$ \\
\hline Previous ipsilateral operation (no.) & $2(1 \%)$ & $2(2 \%)$ & $9(3 \%)$ \\
\hline $\begin{array}{l}\text { American Society of Anesthesiologists } \\
\text { score, (median and range) }\end{array}$ & $3(2-3)$ & $3(2-3)$ & $3(1-3)$ \\
\hline Size of primary tumor* $(\mathrm{cm}$, median and range $)$ & $3.9(0.7-9.4)$ & $3.7(0.7-9.4)$ & $3.6(0.5-20)$ \\
\hline
\end{tabular}

*Variables used for propensity matching. No statistically significant differences were observed in the aforementioned unmatched variables.

who proceeded directly to a thoracotomy for pulmonary resection during this time frame (because of the need for sleeve resections, chest wall resection, Pancoast tumors, etc). The characteristics of these 168 patients are shown in Table 1, which also compares the characteristics of the 106 patients who actually underwent complete CPRL-4 and those of the 318 (3:1 matched) control patients who underwent lobectomy with nerve- and rib-sparing thoracotomy. During the operation, 7 patients were noted after robotic magnified inspection to have unsuspected lesions on the pleura that were biopsy proven as cancer. Thirteen procedures were converted to open (11 lobectomies, 1 segmentectomy, and 1 wedge), and 148 patients underwent a complete robotic resection (106 underwent lobectomy, 26 underwent wedge resection, and 16 underwent segmentectomy).

Table 1 shows that there were no significant differences in the patient characteristics and risk factors between the 2 groups in the unmatched variables. Table 2 illustrates the outcome differences for the 106 patients who underwent CPRL-4 and 318 matched patients who underwent riband nerve-sparing thoracotomy and lobectomy. It shows that there was no statistically significant difference in the total number of lymph nodes removed or in the median number of $\mathrm{N} 2$ or N1 lymph node stations assessed. Additionally, it shows that there was significantly less blood loss $(35 \mathrm{~mL}$ vs $90 \mathrm{~mL} ; P=.03)$, shorter chest tube duration (1.5 vs 3.0 days; $P<.001$ ), shorter hospital stay ( 2 days vs 4 days;
$P=.02)$, lower incidence of morbidity $(27 \%$ vs $38 \%$; $P=.05)$, and lower 3 -week postoperative pain score $(2.5$ vs $4.4 ; P=.04)$ in the CPRL-4 group than in the open group.

Postoperative morbidities included transient atrial fibrillation in 12 patients (all were successfully treated with calcium-channel blockers and discharged home in sinus rhythm), air leak in 10 patients ( 3 patients were discharged home on postoperative day 3 with a chest tube and with a digital air leak system), chylothorax in 2 patients (both patients successfully treated with a medium-chain triglyceride diet), pneumothorax after chest tube removal in 2 patients (both patients required replacement of the chest tube, and these tubes were removed within 48 hours), subcutaneous emphysema in 6 patients (treated by adding suction to the chest tubes), and respiratory distress requiring reintubation in 1 patient (who recovered within 1 week and was discharged home on postoperative day 7).

A significantly higher average mental quality of life score was observed 3 weeks postoperatively in patients who underwent the CPRL-4 robotic lobectomy relative to patients who underwent an open rib- and nervesparing lobectomy (53.5 vs $40.3 ; P<.001$, respectively). A similar trend was observed for physical quality of life score at 3 weeks ( 40.1 vs $34.1 ; P=.07$, respectively); however, the latter difference did not achieve a statistical significance. The 4-month postoperative mental and 
TABLE 2. Comparison of the outcomes of the 106 patients who underwent completely portal robotic lobectomy with 4 robotic arms compared with 318 patients (matched controls) who underwent open lobectomy with rib- and nerve-sparing technique

\begin{tabular}{|c|c|c|c|}
\hline & $\begin{array}{l}\text { Robotic operation } \\
\qquad(\mathbf{N}=106)\end{array}$ & $\begin{array}{l}\text { Rib- and nerve-sparing } \\
\text { thoracotomy }(\mathrm{N}=318)\end{array}$ & $P$ value \\
\hline Estimated blood loss $(\mathrm{mL}$, median $\pm \mathrm{SD})$ & $30 \pm 26$ & $90 \pm 22$ & .03 \\
\hline Operative time ( $h$, median $\pm \mathrm{SD})$ & $2.2 \pm 1.0$ & $1.5 \pm 0.8$ & $<.001$ \\
\hline No. of mediastinal (N2) lymph node stations removed (median) & 5 & 5 & $>.999$ \\
\hline No. of mediastinal (N2) lymph nodes removed (median) & 12 & 11 & .906 \\
\hline No. of N1 lymph node stations removed (median) & 3 & 3 & $>.999$ \\
\hline No. of N1 lymph node removed (median) & 5 & 4 & .89 \\
\hline Chest tube duration ( $\mathrm{d}$, median and range) & $1.5(1-6)$ & $3.0(1-67)$ & $<.001$ \\
\hline Hospital stay (d, median and range) & $2.0(1-7)$ & $4.0(1-67)$ & .01 \\
\hline Morbidity (no.) & $28(27 \%)$ & $120(38 \%)$ & .05 \\
\hline Operative mortality (no.) & 0 & $11(3 \%)$ & .11 \\
\hline Verbal pain score $3 \mathrm{wk}$ postoperatively (median and range) & $2.5(0-7)$ & $4.4(0-8)$ & .04 \\
\hline
\end{tabular}

physical quality of life scores did not differ significantly between the 2 groups.

Thirteen patients had conversion to open procedures during robotic pulmonary resection (11 during CPRL-4 and 1 each during wedge resection and segmentectomy). The reasons for conversions were as follows: 4 patients had invasive tumors ( 2 into the other lobe, 1 into the pericardium, and 1 into the chest wall), there were time limitations in 4 cases (all in our first 15 operations), 3 patients had calcified lymph nodes on the pulmonary artery or bronchus that precluded safe dissection, and 2 patients had all other phases of the lobectomy completed, but the pulmonary vein in 1 case and a bronchus in the other could not be safely taken with the stapler.

Two patients had conversion from a robotic approach to thoracotomy because of vascular injury and bleeding. One patient was undergoing a left upper lobectomy, and there was an injury to the apical anterior trunk of the pulmonary artery while a vessel loop was being placed around it. The area was first packed robotically and confirmed by a VATS camera to be hemostatic; the robot was then undocked, and a thoracotomy was performed in a nonurgent manner. The lobectomy was completed, and the patient did well and went home on postoperative day 4 without the need for any blood transfusions. The second patient was undergoing a posterior segmentectomy of the right upper lobe, and after safe ligation of the posterior segmental artery and bronchus, there was significant bleeding during completion of the operation by stapling the lung parenchyma. This bleeding necessitated a thoracotomy. This patient went home on postoperative day 6 without any complications or the need for blood transfusion, despite poor pulmonary function.

Results of CPRL-4 after technical modifications show a trend toward reduction in median operative time, as shown in Figure 2, and reduction in conversion rate (12 of the first 62 operations [19\%] and 1 in the last $106[1 \%] ; P<.001)$.
The significant technical changes made were as follows: addition of the fourth robotic arm posterior and use of a $5-\mathrm{mm}$ port so that the surgeon could retract the lung for himself or herself; placement of a vessel loop around the artery, vein, bronchus, and fissures to help guide the stapler; the removal of the tumor above the diaphragm; and the use of carbon dioxide insufflation.

\section{DISCUSSION}

The results of this study show that robotic lobectomy is safe. The surgeon can achieve an R0 resection in patients with lung cancer, even those with large tumors (up to 9.4 $\mathrm{cm}$ in this series). In addition, an outstanding mediastinal and hilar lymph node resection is achievable. The median number of $\mathrm{N} 2$ mediastinal lymph node stations removed was 5: stations $2 \mathrm{R}, 4 \mathrm{R}, 7,8$, and 9 in the right side of the chest and stations 5, 6, 7,8, and 9 in the left side of the chest. There also was a median of $3 \mathrm{~N} 1$ hilar lymph nodes removed (stations 10, 11, and 12). Important limitations, however, include and are not limited to cost concerns, the learning

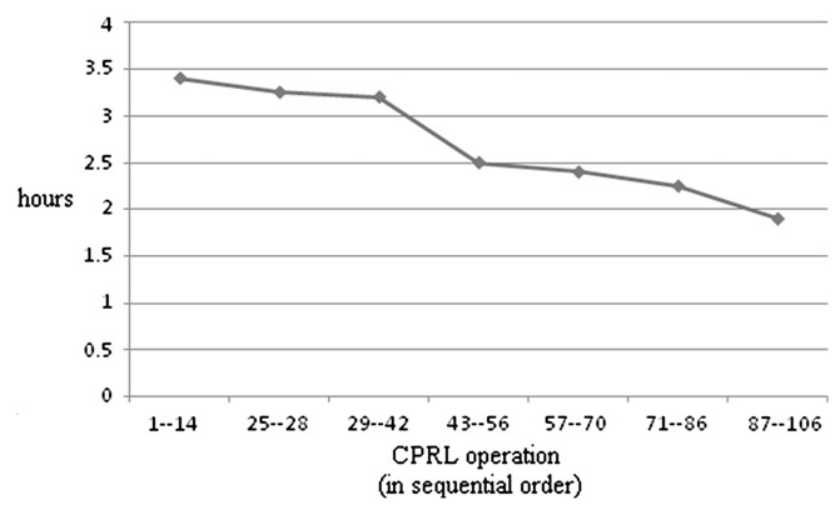

FIGURE 2. Median operative times (skin incision to skin closure) for completed completely portal robotic lobectomy with 4 robotic arms (CPRL) with time in sequential order. 
curve required by the entire team, the inability to palpate the lung, and the inability to obtain adequate robotic time in many hospitals.

This study represents our first 14 months of experience with robotic pulmonary resection. Our initial conversion rate was high (12 of the first 62 operations vs only 1 in the last 106). Importantly, all conversions were elective except 2 , and both of those patients did well and received no blood transfusion. The causes for the high initial conversion rate are multifactorial. First, we were developing our new CPRL-4 procedure, so various port placements, instruments, and robot positionings were tried. Second, this was a consecutive series of patients, not the more typical series of hand-picked patients with small peripheral lesions. Third, we intentionally set a time limit of 4 hours for the operation. This was done not only to protect patients from long anesthetic times and to prevent pulmonary edema ${ }^{20}$ in the dependent lung but also to maintain the confidence of the entire operating room staff and to limit our frustration with learning an entirely new way to operate. Once the operative technique had solidified, only 1 of the last 106 patients had conversion to an open procedure, even though many patients had previous thoracotomy, large tumors, and preoperative radiation therapy and chemotherapy.

Reasons to perform robotic or VATS operations are several-fold. New provocative data suggest immunologic benefits to a minimally invasive, non-rib spreading operation, which may lead to improved survival for patients with non-small cell lung cancer. ${ }^{21-24}$ These important data (and the fact that we were unsatisfied with our lymph node dissection during VATS) provided the impetus for us even to try robotic pulmonary resection. Studies show that patients who undergo minimally invasive lobectomy by VATS have shorter hospital stay, less morbidity, quicker recovery, quicker chest tube removal, and the better delivery of adjuvant chemotherapy. ${ }^{25}$ We have argued and still believe that that many of the advantages of minimally invasive surgery are related more to the surgeon's mindset than to the chosen operative technique. Improved 5-year survival and lessened morbidity, however, are important reasons for all surgeons to adopt minimally invasive techniques. These facts make the fervent debate between thoracic surgeons regarding robotic lobectomy versus VATS lobectomy seem trivial and silly. Our ultimate goal as general thoracic surgeons should be the same: a minimally invasive, non-rib spreading approach for patients with lung cancer that still provides all the oncologic benefits of surgery by thoracotomy.

This study enrolled many patients who probably would not have been offered VATS lobectomy at most institutions. Most VATS studies select patients with small T1 or T2 N0 M0 (usually $<3 \mathrm{~cm}$ ) lesions. ${ }^{26,27}$ This is because the tumor is removed though a 3- to 4-cm access incision most frequently made between ribs 4 and 5 or 5 and 6 . In contrast, a CPRL-4 uses a lower rib space for the removal of tumors (one that VATS surgeons could use as well) between ribs 9 and 10 anteriorly. This permits the removal of larger tumors (as large as $9.4 \mathrm{~cm}$ in this series). Robotic surgery, however, clearly takes longer to set up (more trocars and ports are used, and there is necessary docking time), has higher initial capital costs, provides no lung palpation, and involves more specialized equipment than VATS.

Previous reports have demonstrated the safety of robotic pulmonary resection, although they have used very different operative techniques. The technical differences are important to the surgeon. Veronesi and associates ${ }^{20}$ in 2010 recently reported the safety of a 4-arm robotically assisted (not completely portal) lobectomy (with a 3- to 4-cm access incision such as used by VATS surgeons) in 54 patients. Ninan and coworkers ${ }^{28}$ in 2010 reported the effectiveness of a completely portal robotic lobectomy with 3 arms in 74 patients. Gharagozloo and colleagues ${ }^{29}$ in 2009 reported outcomes with a hybrid technique. Thus the literature shows that robotic pulmonary resection is safe and oncologically sound, allowing R0 resection with excellent lymph node removal. Similar to VATS, there are several ways that robotic resection can be performed. An international committee has been formed and is writing a consensus paper regarding agreed upon nomenclature and definitions for the different types of robotic surgery (ie, robotically assisted, completely portal, etc).

The adoption of the robot for pulmonary resection will depend on several factors: the availability of the robotic platform to the thoracic surgeon; the true cost of the operation; the measured and perceived benefits to the patient, hospital, and surgeon; and the time it takes to perform the operation. Most important, however, may be the surgeon's current enthusiasm for the VATS lobectomy that he or she performs: minimally invasive surgery is unequivocally our future. If a team is already adroit with VATS lobectomy and believes that the lymph node dissection being attained is adequate, the desire to add robotic pulmonary resection to the armamentarium will be low. If lymph node dissection during VATS lobectomy is, however, suboptimal and difficult to teach, as in our experience, then the desire for robotic techniques will probably be high.

The strengths of this study are as follows: there was only 1 surgeon who performed all the open and robotic operations, and it was a consecutive series as opposed to highly selected patients. The limitations of this study include the following: it was single institutional; the procedure underwent modifications during the first 20 operations; and patients were told that the procedure was new and "less invasive," and this information may have biased the patients during their reporting of their pain scores and quality of life surveys.

In conclusion, this study demonstrates the safety, efficacy, complete lymph node removal, and improved quality 
of life of a CPRL-4 operation for patients with non-small cell lung cancer. Technical modifications are possible and can lead to decreased operative times and improved teachability, as well as decreasing patient morbidity and surgeon frustration during the learning curve. Even though hospitals are acquiring more robots for other specialties in addition to thoracic surgery, the capital cost, service contract costs, and equipment costs must be carefully considered and studied. Patient selection is critical, especially during the learning curve. In our opinion, there are few to any achievable benefits of using a robotic system rather than VATS when performing a sympathotomy for patients with hyperhidrosis or a pulmonary wedge resection for tissue diagnosis for patients with interstitial lung disease. Implementation of a robotic system for the resection of mediastinal tumor or for thymectomy in patients with myasthenia gravis is, however, of obvious potential utility. Although further studies are needed, the explosion of the interest in robotics in general thoracic surgery is real. Academic centers must unite and organize to ensure the proper training of surgeons in robotic techniques to protect patients and surgeons and to form an agreed upon international nomenclature.

\section{References}

1. Carreyrou J. Surgical robot examined in injuries. The Wall Street Journal. 2010 May 4;Sect. A:1.

2. Barbash GI, Glied G. New technology and health care costs-the case of robotassisted surgery. N Engl J Med. 2010;363:701-4.

3. Ashton RC Jr, Connery CP, Swistel DG, DeRose JJ Jr. Robot-assisted lobectomy. J Thorac Cardiovasc Surg. 2003;126:292-3.

4. Morgan JA, Ginsburg ME, Sonett JR, Argenziano M. Thoracoscopic lobectomy using robotic technology. Heart Surg Forum. 2003;6:E167-9.

5. Bodner J, Wykypiel H, Wetscher G, Schmid T. First experiences with the da Vinci operating robot in thoracic surgery. Eur J Cardiothorac Surg. 2004;25: 844-51.

6. Yamada K, Kato S. Robot-assisted thoracoscopic lung resection aimed at solo surgery for primary lung cancer. Gen Thorac Cardiovasc Surg. 2008;56:292-4.

7. Cerfolio RJ, Bryant AS. Survival of patients with unsuspected N2 (stage IIIA) nonsmall-cell lung cancer. Ann Thorac Surg. 2008;86:362-7.

8. Bryant AS, Rudemiller K, Cerfolio RJ. The 30- versus 90- day operative mortality after pulmonary resection. Ann Thorac Surg. 2010;89:1717-23.

9. Cerfolio RJ, Bryant AS, Maniscalco LM. A non-divided intercostal muscle flap further reduces pain of thoracotomy: a prospective randomized trial. Ann Thorac Surg. 2008;85:1901-7.

10. Cerfolio RJ, Bryant AS, Patel B, Bartolucci AA. Intercostal muscle flap reduces the pain of thoracotomy: a prospective randomized trial. $J$ Thorac Cardiovasc Surg. 2005;130:987-93.

11. Cerfolio RJ, Bryant AS, Bass CS, Bartolucci AA. A prospective, double-blinded, randomized trial evaluating the use of preemptive analgesia of the skin before thoracotomy. Ann Thorac Surg. 2003;76:1055-8.

12. Cerfolio RJ, Price TN, Bryant AS, Sale Bass C, Bartolucci AA. Intracostal sutures decrease the pain of thoracotomy. Ann Thorac Surg. 2003;76:407-12.

13. Allama AM. Intercostal muscle flap for decreasing pain after thoracotomy: a prospective randomized trial. Ann Thorac Surg. 2010;89:195-9.

14. Sakakura N, Usami N, Taniguchi T, Kawaguchi K, Okagawa T, Yokoyama M, et al. Assessment of long-term postoperative pain in open thoracotomy patients: pain reduction by the edge closure technique. Ann Thorac Surg. 2010;89: 1064-70.

15. Ibrahim HN, Foley R, Tan L, Rogers T, Bailey RF, Guo H, et al. Long-term consequences of kidney donation. N Engl J Med. 2009;360:459-69.

16. Ware J Jr, Kosinski M, Keller SD. A 12-Item Short-Form Health Survey: construction of scales and preliminary tests of reliability and validity. Med Care. 1996;34:220-33.
17. Ghanta RK, Shekar PS, McGurk S, Rosborough DM, Aranki SF. Nonelective cardiac surgery in the elderly: is it justified? J Thorac Cardiovasc Surg. 2010;140: 103-9.

18. Boodhwani M, Rubens F, Wozny D, Rodriguez R, Nathan HJ. Effects of sustained mild hypothermia on neurocognitive function after coronary artery bypass surgery: a randomized, double-blind study. J Thorac Cardiovasc Surg. 2007;134: 1443-52.

19. Rosenbaum PR, Rubin DB. The central role of the propensity score in observational studies for causal effects. Biometrika. 1983;70:41-55.

20. Veronesi G, Galetta D, Maisonneuve P, Melfi F, Schmid RA, Borri A, et al. Fourarm robotic lobectomy for the treatment of early-stage lung cancer. $J$ Thorac Cardiovasc Surg. 2010;140:19-25.

21. Mahtabifard A, DeArmond DT, Fuller CB, McKenna RJ Jr. Video-assisted thoracoscopic surgery for stage I lung cancer. Thorac Surg Clin. 2007;17:223-31.

22. Whitson BA, Groth SS, Duval SS, Swanson SJ, Maddaus MA. Surgery for early stage non-small cell lung cancer: a systematic review of the video-assisted thoracoscopic surgery versus thoracotomy approach to lobectomy. Ann Thorac Surg. 2008;86:2008-18.

23. Yan TD, Black D, Bannon PG, McCaughan M. Systematic review and metaanalysis of randomized and non-randomized trials on safety and efficacy of video-assisted thoracic surgery lobectomy for early stage non-small cell lung cancer. J Clin Oncol. 2009;27:2553-62.

24. Flores RM, Ihekweasu UN, Rizk N, Dycoco BA, Bains MS, Downey RJ, et al Patterns of recurrence and incidence of second primary tumors after lobectomy by means of video-assisted thoracoscopic surgery (VATS) versus thoracotomy for lung cancer. J Thorac Cardiovasc Surg. 2011;141:59-64.

25. Onaitis MW, Petersen PR, Balderson SS, Toloza E, Burfeind WR, Harpole DH Jr, et al. Thoracoscopic lobectomy is a safe and versatile procedure: experience with 500 consecutive patients. Ann Surg. 2006;244:420-5.

26. McKenna RJ Jr, Mahtabifard A, Pickens A, Kusuanco D, Fuller CB. Fast-tracking after video-assisted thoracoscopic surgery lobectomy, segmentectomy, and pneumonectomy. Ann Thorac Surg. 2007;84:1663-8.

27. Hartwig MG, D'Amico TA. Thoracoscopic lobectomy: the gold standard for early-stage lung cancer? Ann Thorac Surg. 2010;89:S2098-101.

28. Ninan M, Dylewski MR. Total port-access robot assisted pulmonary lobectomy without utility thoracotomy. Eur J Cardiothorac Surg. 2010;38:231-2.

29. Gharagozloo F, Margolis M, Tempesta B, Strother E, Najam F. Robot-assisted lobectomy for early-stage lung cancer: report of 100 cases. Ann Thorac Surg. 2009; 88:380-4.

\section{APPENDIX 1. Operative Description of CPRL-4}

As shown in Figure 1, this new technique can be briefly summarized. The pleural space is entered over the top of the 7th rib with a 5-mm port in the midaxillary line, or as anteriorly as possible, and guided by a $5-\mathrm{mm}$ scope. A 5-mm VATS camera is used to ensure entry into the pleural space, and warmed carbon dioxide is insufflated to drive the diaphragm inferiorly. This incision will eventually be enlarged to allow a $12-\mathrm{mm}$ port, and it will serve as robotic arm 1 (for right-sided operations). A paravertebral block is performed posteriorly with a local anesthetic and a 21gauge needle from ribs 3 to 11 . The needle is used to help select the ideal location for the second incision, the most posterior incision. The location chosen is 2 ribs below the major fissure and as far posterior in the chest as possible, just anterior to the spinal processes of the vertebral body. A small 5-mm incision is made, and a 5-mm reusable metal da Vinci trocar is placed. This will be the position for robotic arm 3 . The next few incisions are carefully planned and marked on the skin before they are made. Ten centimeters anterior to the most posterior incision and along the same rib (most commonly rib 8), a third incision is planned. It is an incision for an 8-mm port, and its trocar is an 8-mm 
metal reusable da Vinci trocar that will be docked with robotic arm 2. A fourth incision is marked on the skin and again planned but not made $9 \mathrm{~cm}$ anterior to this port, along the same rib as shown in Figure 1. This will eventually be used for the robotic camera. A 12-mm plastic disposable port is used for the 12-mm camera, and if the $8-\mathrm{mm}$ camera is used, an $8-\mathrm{mm}$ metal reusable trocar is placed. Before these 2 incisions are made, a small 21 -gauge needle is used to identify the most anteriorly inferior aspect of the chest that is just above the diaphragmatic fibers. This incision will have a $15-\mathrm{mm}$ port and serve as the access port. A plastic disposable trocar is used. No robotic arms are attached to the trocar that is placed in this incision. This incision is carefully planned. It is made just above the diaphragm, as anterior and inferior as possible and, importantly, to be in between the ports used for robotic arm 1 and the camera. The access port can alternatively be more posterior if anatomy dictates between the camera and robotic arm 2 . It should be 2 or 3 ribs lower than these 2 ports. This affords room for the bedside assistant to work. Once these incisions have been carefully planned and their locations have been confirmed, they are made and the appropriate trocars are placed. Finally, the initial $5-\mathrm{mm}$ anterior port that was made first and used to introduce the VATS camera to identify the internal landmarks is then dilated to a $12-\mathrm{mm}$ doublecannulated port for robotic arm 1 . The robot is driven over the patient's shoulder on a $15^{\circ}$ angle and attached to the 4 ports. In general, only 4 robotic instruments were used for all these operations: the Cadierre grasper, a 5-mm bowel grasper (used exclusively through the most posterior port that is attached to robotic arm 3, which serves as a retractor of the lung), the Maryland forceps, and a cautery spatula. 